\title{
Physical Parameter Identification of a Viscoelastic Chain from Time Response Data
}

\author{
Y.M. Ram* and G.Z. Voyiadjis** \\ *Department of Mechanical Engineering \\ Louisiana State University \\ Baton Rouge, LA 70803, USA \\ ram@me.lsu.edu \\ **Boyd Professor \\ Department of Civil and Environmental Engineering \\ Louisiana State University \\ Baton Rouge, LA 70803, USA \\ voyiadjis@eng.lsu.edu
}

\begin{abstract}
It is shown that an $n$ degree-of-freedom chain of springs and viscous dampers, which is subject to a constant unit load, may be reconstructed uniquely from $2 n$ time dependent displacement data at the chain's end. This may effectively in application allow one to obtain a reduction in stiffness due to damage in structural components such as pavements and fuselages.
\end{abstract}

\section{INTRODUCTION}

Consider an $n$ degree-of-freedom chain consisting of linear springs $\boldsymbol{\kappa}_{j}>0$ and viscous dampers $\gamma_{j}>\mathbf{0}$, $i=1,2, \ldots, n$, arranged as shown in Figure 1(a). Let $x_{j}$ denote the time dependent displacement of the $j$ th node of the chain from its static equilibrium position.

This system may represent an elastic structural body that rests on a platform with specific frictional characteristics between the structure and the platform. These frictional effects are described in terms of the dashpot characteristics in this system.

Suppose that a unit force $f=1$ is applied to the free end of the chain. Then the equations of motion for the nodes can be written in the following matrix form

$$
\mathbf{G} \mathbf{x}+\mathbf{K x}=\mathbf{f},
$$




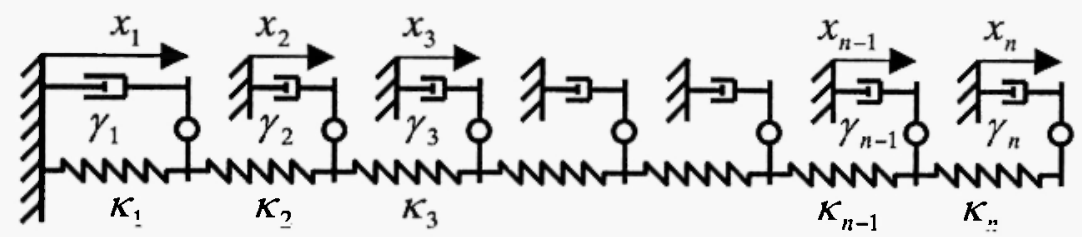

(a) Free spring-damper chain

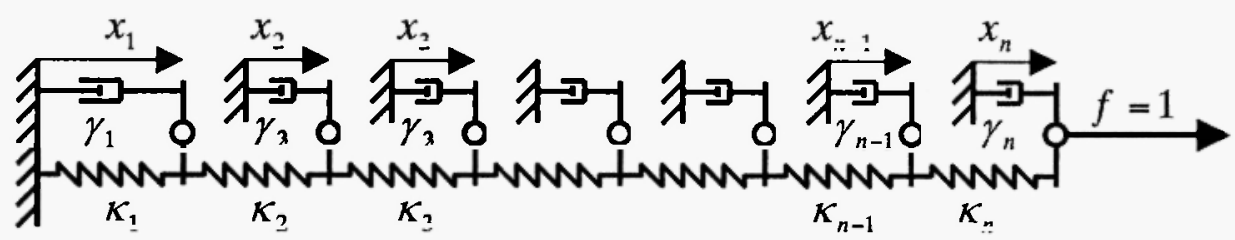

(b) Forced system

Fig. 1: Description of system models.

where

$$
\mathbf{G}=\operatorname{diag}\left\{\begin{array}{llll}
\gamma_{1} & \gamma_{2} & \cdots & \gamma_{n}
\end{array}\right\},
$$

$$
\mathbf{K}=\left[\begin{array}{ccccc}
\kappa_{1}+\kappa_{2} & -\kappa_{2} & & & \\
-\kappa_{2} & \kappa_{2}+\kappa_{3} & -\kappa_{3} & & \\
& \ddots & \ddots & \ddots & \\
& & \cdots \kappa_{n-1} & \kappa_{n-1}+\gamma_{n} & -\kappa_{n} \\
& & & -\kappa_{n} & \kappa_{n}
\end{array}\right]
$$

$$
\mathbf{x}=\left(\begin{array}{llll}
x_{1} & x_{2} & \cdots & x_{n}
\end{array}\right)^{T},
$$

and $\mathrm{f}$ is the $n$th unit vector,

$$
\mathbf{f}=\mathbf{e}_{n}=\left(\begin{array}{llll}
0 & 0 & \cdots & 1
\end{array}\right)^{T} .
$$

It is shown that the system may be reconstructed uniquely from $2 n$ time displacement data $x_{n}\left(t_{k}\right)$, $t_{k}=k \Delta t, k=0,2, \ldots, 2 n-1$. The reconstruction process involves two well-known procedures, one of fitting an exponential function model to the measured data, and the other of evaluating a tridiagonal system model from its spectrum. These procedures are documented in Section 3 and Section 4, respectively. A small detailed example demonstrating the entire identification process is given in Section 5 , and the paper is summarized in Section 6. 


\section{THE SYSTEM RESPONSE}

Let $\lambda_{j}$ and $\mathbf{u}_{j} \neq \mathbf{0}$ be the $j$ th eigenvalue and its associated eigenvector of the generalized eigenvalue problem

$$
(\mathbf{K}-\lambda \mathbf{G}) \mathbf{u}=\mathbf{o} .
$$

It is well known, e.g. Golub and Van Loan, 1986, that the eigenvalues $\lambda_{j}$ of (6) are positive and distinct whenever $\kappa_{j}>0$ and $\gamma_{j}>0$, since the eigenvalue problem (6) is in this case congruently equivalent to a non-singular Jacobi system with a weakly dominant diagonal. It thus follows that the response of the system (1) is given by

$$
x(\mathbf{t})=g+\sum_{j=1}^{\mathbf{n}} a_{j} u_{j} e^{-\lambda_{j} t},
$$

where $\mathbf{g}$ is a constant vector,

$$
\mathbf{g}=\mathbf{K}^{-1} \mathbf{e}_{n},
$$

describing the steady state displacement of the various degrees of freedom. If the system starts to move from rest then $\mathbf{x}(0)=\mathbf{o}$ and we have

$$
\mathbf{a}=-\mathbf{U}^{-1} \mathbf{g}=-(\mathrm{KU})^{-1} \mathbf{e}_{\mathrm{n}},
$$

where

$$
\mathbf{a}=\left(\begin{array}{llll}
a_{1} & a_{2} & \cdots & a_{n}
\end{array}\right)^{T},
$$

and

$$
\mathrm{U}=\left[\begin{array}{llll}
\mathbf{u}_{1} & \mathbf{u}_{2} & \cdots & \mathbf{u}_{n}
\end{array}\right]
$$

The response of the free end of the chain is thus

$$
x_{n}(t)=\mathbf{e}_{n}^{T} \mathbf{x}=\mathbf{e}_{n}^{T} \mathbf{g}+\sum_{j=1}^{n} a_{j} \mathbf{e}_{n}^{T} \mathbf{u}_{j} e^{-\lambda_{j} t},
$$

or 


$$
x_{n}(t)=b_{0}+\sum_{j=1}^{n} b_{j} e^{-\cdot \lambda_{j} t}
$$

with the obvious definitions of $b_{j}, j=0,1,2, \ldots, n$.

Knowing $x_{n}(t)$, the problem under consideration is of determining the physical parameters $\kappa_{j}, \gamma_{j}$, $j=1,2, \ldots, n$, of the model. We first determine in the next section the mathematical parameters $b_{l}$ and $\lambda_{j}$ from the measured data, then show in Section 4 how to extract the physical parameters $\kappa_{j}, \gamma_{j}$ from $b_{j}$ and $\lambda_{j}$

\section{EVALUATING THE METHEMATICAL PARAMETERS}

Denote

$$
h(t) \equiv x_{n}(t)
$$

and assume that $x_{n}(t)$ has been sampled with constant sampling rate $\Delta t$. We define

$$
h_{k} \equiv h(k \Delta t), k=0,1, \ldots, m,
$$

so that (13) can be written in the form

$$
h_{k}=b_{0}+\sum_{j=1}^{n} b_{j} e^{-\lambda, k \Delta t}, \quad k=0,1, \ldots, m,
$$

by virtue of (14) and (15). Note that the condition $x(0)=0$ implies that

$$
b_{0}=-\sum_{j=1}^{n} b_{j} \text {, }
$$

where $b_{0}$ is the steady state displacement of $x_{n}$. We further denote $h_{k}=h_{k+1}-h_{k}$ and obtain

$$
h_{k}=\sum_{j=1}^{n} \xi_{j} e^{-\lambda_{j} k \Delta t}, k=0,1, \ldots, m-1
$$

where

$$
\xi_{j}=b_{j}\left(e^{-\lambda_{j} \Delta t}-1\right)
$$


The problem of determining $\lambda_{j}$ and $\xi_{j}$, knowing $\Delta t$ and $m=2 n$ data values $h_{k}$, is thus the well known exponential fitting problem, which can be solved for example by Prony's method (Hamming, 1987). Following Hamming we determine the coefficients $c_{j}, j=0,1, \ldots, n-1$ of the monic polynomial

$$
L(z)=\prod_{j=1}^{n}\left(z-z_{j}\right)=\sum_{j=1}^{n} c_{j} z^{j}, c_{n}=1,
$$

via the solution of the linear system

$$
\left[\begin{array}{ccccc}
h_{0} & h_{1} & h_{2} & \cdots & h_{n-1} \\
h_{1} & h_{2} & h_{3} & \cdots & h_{n} \\
h_{2} & h_{3} & h_{4} & \cdots & h_{n+1} \\
\vdots & \vdots & \vdots & \vdots & \vdots \\
h_{n-1} & h_{n} & h_{n+1} & \cdots & h_{2 n-2}
\end{array}\right]\left(\begin{array}{c}
c_{0} \\
c_{1} \\
c_{2} \\
\vdots \\
c_{n-1}
\end{array}\right)=\left(\begin{array}{c}
-h_{n} \\
-h_{n+1} \\
-h_{n+2} \\
\vdots \\
-h_{2 n-1}
\end{array}\right)
$$

The roots $z_{j}$ of $L(z)$ generally determine the poles $\lambda_{j}$ via

$$
\lambda_{j}=-\frac{\ln \left|z_{j}\right|+i \arg z_{j}}{\Delta t}, i=\sqrt{-1} .
$$

In our case, whenever the data are sympathetic with the model, the roots $z_{j}$ are real positive and $\lambda_{j}$ is determined uniquely from

$$
\lambda_{j}=-\frac{1}{\Delta t} \ln z_{j}
$$

Knowing $\lambda_{j}, j=1,2, \ldots, n$, the coefficients $\zeta_{j}$ in (18) are determined by the solution of the following system:

$$
\left[\begin{array}{cccc}
1 & 1 & \cdots & 1 \\
e^{-\lambda_{1} \Delta t} & e^{-\lambda_{2} \Delta t} & \cdots & e^{-\lambda_{n} \Delta t} \\
\vdots & \vdots & \cdots & \vdots \\
e^{-\lambda_{1}(n-1) \Delta t} & e^{-\lambda_{2}(n-1) \Delta t} & \cdots & e^{-\lambda_{n}(n-1) \Delta t}
\end{array}\right]\left(\begin{array}{c}
\xi_{1} \\
\xi_{2} \\
\vdots \\
\xi_{n}
\end{array}\right)-\left(\begin{array}{c}
h_{0} \\
h_{1} \\
\vdots \\
h_{n-1}
\end{array}\right)
$$

At this stage where the system parameters $\xi_{j}, \lambda_{j}, j=1,2, \ldots, n$, are known, the coefficients $b_{j}$, and in particular the steady state displacement at the right end of the chain $b_{0}$ can be determined from (17) and (19). Then Laplace transform of (18) yields 


$$
H(s)=\frac{b_{0}}{s}+\sum_{j=1}^{n} \frac{b_{j}}{s+\lambda_{j}}=\frac{P(s)}{s \prod_{j=1}^{n}\left(s+\lambda_{j}\right)}
$$

where, by virtue of (17) $P(s)$ is a polynomial of degree $n-1$

$$
P(s)=\eta \prod_{j=1}^{n-1}\left(s+\mu_{j}\right),
$$

where $\eta$ is some constant. The root $-\mu_{j}, j=1,2, \ldots, n-1$ of $P(s)$ are the zeros of the transfer function $H(s)$. They are the roots of the characteristic polynomial

$$
\operatorname{det}(\mathbf{K}-\lambda \mathbf{G})=0
$$

where $\mathbf{K}$ and $\mathbf{G}$ are the $(n-1) \times(n-1)$ leading principal submatrices of $\mathbf{K}$ and $\mathbf{G}$,

$$
\mathbf{K}=\left[\begin{array}{ccccc}
\kappa_{1}+\kappa_{2} & -\kappa_{2} & & & \\
-\kappa_{2} & \kappa_{2}+\kappa_{3} & -\kappa_{3} & & \\
& \ddots & \ddots & \ddots & \\
& & -\kappa_{n-2} & \kappa_{n-2}+\gamma_{n-1} & -\kappa_{n-1} \\
& & & -\kappa_{n-1} & \kappa_{n-1}+\kappa_{n}
\end{array}\right],
$$

and

$$
\mathbf{G}=\operatorname{diag}\left\{\begin{array}{llll}
\gamma_{1} & \gamma_{2} & \cdots & \gamma_{n-1}
\end{array}\right\}
$$

It is well known, see e.g., Golub and Van Loan, 1986, that the $\mu_{i}, j=1,2, \ldots, n-1$, are all positive, and moreover, they interlace $\lambda_{j}, j=1,2, \ldots, n$,

$$
\lambda_{1}<\mu_{1}<\lambda_{2}<\cdots<\lambda_{n-1}<\mu_{n-1}<\lambda_{n}
$$

It will now be shown how to evaluate the physical dampers $\gamma_{j}$ and the springs $\kappa_{j}$ knowing the poles $-\lambda_{j}$ and zeros $-\mu_{j}$ of $H(s)$, and the steady state displacement $b_{0}$ of $x_{n}$. 


\section{EVALUATING THE PHYSICAL PARAMETERS OF THE MODEL}

We are now in a position where $n$ positive poles $\lambda_{j}, n-1$ positive zeros $\mu$, satisfying the interlacing property (28), and the steady state displacement $b_{0}$, are known. The problem of determining the physical parameters $\gamma_{j}$ and $\kappa_{j}, j=1,2, \ldots, n$, from these data is similar to the problem of reconstructing a chain of massspring system from spectral data, which was first addressed by Gantmakher and Krein, 1961. The solution presented here is based on the Lanczos method, applied by de Boor and Golub, 1978, for reconstructing a tridiagonal matrix from spectral data.

A matrix $\mathbf{A}$ is first defined

$$
A=G^{-1 / 2} \mathbf{K G}^{-1 / 2}
$$

where

$$
\mathbf{G}^{-1 / 2}=\operatorname{diag}\left\{\begin{array}{llll}
\gamma_{1}^{-1 / 2} & \gamma_{2}^{-1 / 2} & \cdots & \gamma_{n}^{-1 / 2}
\end{array}\right\}
$$

The matrix $\mathbf{A}$ is symmetric tridiagonal

$$
\mathbf{A}=\left[\begin{array}{ccccc}
\alpha_{1} & \beta_{1} & & & \\
\beta_{1} & \alpha_{1} & \beta_{2} & & \\
& \ddots & \ddots & \ddots & \\
& & \beta_{n-2} & \alpha_{n-1} & \beta_{n-1} \\
& & & \beta_{n-1} & \alpha_{n}
\end{array}\right]
$$

with positive diagonal elements $\alpha_{l}$ and negative off diagonal $\beta_{j}$. Its eigenvalues are $\lambda_{j}, j=1,2, \ldots, n$. If the last row and column of $\mathbf{A}$ are omitted, then the resulting matrix,

$$
\hat{\mathbf{A}}=\left[\begin{array}{ccccc}
\alpha_{1} & \beta_{1} & & & \\
\beta_{1} & \alpha_{1} & \beta_{2} & & \\
& \ddots & \ddots & \ddots & \\
& & \beta_{n-3} & \alpha_{n-2} & \beta_{n-2} \\
& & & \beta_{n-2} & \alpha_{n-1}
\end{array}\right]
$$

has eigenvalues $\mu_{j=.} j=1,2, \ldots, n-1$. These are the data obtained in the previous section.

Denote the spectral decomposition of $\mathbf{A}$ by 
$\mathbf{A V}=\mathbf{\Lambda} \mathbf{V}$

where $\mathbf{V}$ is orthogonal

$$
\mathbf{V}^{T} \mathbf{V}=\mathbf{I},
$$

I is the identity matrix, and $\Lambda$ is diagonal

$$
\Lambda=\operatorname{diag}\left\{\begin{array}{llll}
\lambda_{1} & \lambda_{2} & \cdots & \lambda_{n}
\end{array}\right\}
$$

Then the $n$th row of $\mathrm{V}$ is determined by (de Boor and Golub, 1978),

$$
v_{n j}^{2}=\frac{\prod_{\substack{k=1 \\ k=1}}^{n-1}\left(\lambda_{j}-\mu_{k}\right)}{\prod_{\substack{k=j \\ k \neq j}}^{n-1}\left(\lambda_{j}-\lambda_{k}\right)}, j=1,2, \ldots, n
$$

The last row of (33) can be written as follows:

$$
\begin{gathered}
\beta_{n-1} v_{n-1,1}+\alpha_{n} v_{n 1}=\lambda_{1} v_{n 1} \\
\beta_{n-1} v_{n-1,2}+\alpha_{n} v_{n 2}=\lambda_{2} v_{n 2} \\
\vdots \\
\beta_{n-1} v_{n-1, n}+\alpha_{n} v_{n n}=\lambda_{n} v_{n n} .
\end{gathered}
$$

Multiplying the $j$ th equation of (37) by $v_{i j ;}$ and adding the $n$ equations gives

$$
\alpha_{n}=\sum_{j=1}^{n} \lambda, v_{n j}^{2},
$$

since $\sum_{j=i}^{n} v_{: i j}^{?}=1$ and $\sum_{j-1}^{n} v_{n-1, j} v_{n j}=0$ by virtue of the orthogonality (34). We can therefore determine $\alpha_{n}$ from (38) and (36). Then we obtain the following set of equations from (37)

$$
\begin{gathered}
\left(\alpha_{n}-\lambda_{1}\right)^{2} v_{n 1}^{2}=\beta_{n-1}^{2} v_{n-1,1}^{2} \\
\left(\alpha_{n}-\lambda_{2}\right)^{2} v_{n 2}^{2}=\beta_{n-1}^{2} v_{n-1,2}^{2} \\
\vdots \\
\left(\alpha_{n}-\lambda_{n}\right)^{2} v_{n n}^{2}=\beta_{n-1}^{2} v_{n-1, n}^{2} .
\end{gathered}
$$


Adding these equations we obtain

$$
\beta_{n-1}=-\sqrt{\sum_{j=1}^{n}\left(\alpha_{n}-\lambda_{j}\right)^{2} v_{n j}^{2}}
$$

since $\sum_{i=1}^{n} v_{n-1, j}^{2}=1$. Knowing $\alpha_{n}$ and $\beta_{n-1}$ the $(n-1)$ th row of $\mathbf{V}$ is obtained from (37). The other elements of $\mathrm{A}$ can then be determined successively for $j=n-1, n-2, \ldots, 1$ by following similar process in which $\alpha_{1}$ and $\beta_{j-1}$ are determined by the set of equations

$$
\begin{gathered}
\beta_{j-1} v_{j-1,1}+\alpha_{j} v_{j 1}+\beta_{j} v_{j+1,1}=\lambda_{1} v_{j 1} \\
\beta_{j-1} v_{j-1,2}+\alpha_{j} v_{j 2}+\beta_{j} v_{j+1,2}=\lambda_{2} v_{j 2} \\
\vdots \\
\beta_{j-1} v_{j-1, n}+\alpha_{j} v_{j n}+\beta_{j} v_{j+1, n}=\lambda_{n} v_{j n},
\end{gathered}
$$

with $\beta_{0}=0$, which are obtained from the $j$ th row of (33).

After reconstructing $\mathbf{A}$ the matrices $\mathbf{K}$ and $\mathbf{G}$ are found as follows. Denote

$$
\mathbf{p}=\sqrt{\gamma_{1} / \kappa_{1}}\left(\begin{array}{llll}
1 & 1 & \cdots & 1
\end{array}\right)^{T}
$$

Then multiplying (29) by $\mathbf{G}^{1 / 2} \mathbf{p}$ gives

$$
\mathbf{A G}^{1 / 2} \mathbf{p}=\mathbf{G}^{-1 / 2} \mathbf{K p}
$$

It follows from (2), (3) and (42) that

$$
\mathbf{G}^{-1 / 2} \mathbf{K p}=\mathrm{e}_{1}
$$

where $e_{1}=\left(\begin{array}{llll}1 & 0 & \ldots & 0\end{array}\right)^{T}$. Hence, the vector $y$ determined from

$$
\mathbf{A y}=\mathbf{e}_{1},
$$

where, view of (2) and (42)-(44),

$$
\mathbf{y}=\frac{\sqrt{\gamma_{1}}}{\kappa_{1}}\left(\begin{array}{llll}
\sqrt{\gamma_{1}} & \sqrt{\gamma_{2}} & \cdots & \left.\sqrt{\gamma_{n}}\right)^{T}
\end{array}\right.
$$


The vector $\mathbf{y}$ determines $\mathbf{G}$ up to a scale factor $\gamma_{1} / \kappa_{1}^{2}$. We now show how to determine this factor. It follows from (29) that

$$
\mathbf{K}=\frac{\kappa_{1}^{2}}{\gamma_{1}} \mathbf{Y A Y}
$$

where

$$
\mathbf{Y}=\operatorname{diag}\left\{\begin{array}{llll}
y_{1} & y_{2} & \cdots & y_{n}
\end{array}\right\}
$$

Note that at this stage $\mathbf{A}$ and $\mathbf{Y}$ are known. The steady state deflection $b_{0}$ is related to the flexibility matrix,

$$
b_{0}=\mathbf{e}_{n}^{T} \mathbf{K}^{-1} \mathbf{e}_{n} .
$$

Therefore using (46) we have

$$
b_{0}=\frac{\gamma_{1}}{\kappa_{1}^{2}} \mathbf{e}_{n}^{T} \mathbf{Y}^{-1} \mathbf{A}^{-1} \mathbf{Y}^{-1} \mathbf{e}_{n}
$$

and the scale factor in (47) is

$$
\frac{\sqrt{\gamma_{1}}}{\kappa_{1}}=\sqrt{\frac{b_{0}}{\mathbf{e}_{n}^{T} \mathbf{Y}^{-1} \mathbf{A}^{-1} \mathbf{Y}^{-1} \mathbf{e}_{n}}}
$$

The matrices $\mathbf{G}$ and $\mathbf{K}$ are thus determined by (46) and (47) respectively.

\section{AN EXAMPLE}

Let $n=2$, and suppose that the following data have been measured using the sampling rate $\Delta t=1$ :

$$
h(1)=0.699318, h(2)=1116955, h(3)=1.397648, h(4)=1.588909 .
$$

Then from the definition $h_{k}=h_{k+1}-h_{k}$ with $h(0)=0$ we obtain

$$
h(0)=0.699318, h(1)=0.417637, h(2)=0.280693, h(3)=0.191261
$$


Equation (21)

$\left[\begin{array}{ll}0.699317 & 0.417637 \\ 0.417637 & 0.280693\end{array}\right]\left(\begin{array}{l}c_{0} \\ c_{1}\end{array}\right)=\left(\begin{array}{l}-0.280693 \\ -0.191261)\end{array}\right)$,

then yields

$c_{0}=0.049787, c_{1}=-0.755464$.

The characteristic polynomial (20) is

$$
L(z)=z^{2}-0.755464 z+0.049787,
$$

and its roots are

$$
z_{1}=0.682518, z_{2}=0.072946
$$

The mapping (22) from $z_{j}$ to $s_{i}$ gives

$$
\lambda_{1}=0.381966, \lambda_{2}=2.618034 \text {. }
$$

The system of equation (21)

$$
\left[\begin{array}{cc}
1 & 1 \\
0.682518 & 0.072946
\end{array}\right]\left(\begin{array}{l}
\xi_{1} \\
\xi_{2}
\end{array}\right)=\left(\begin{array}{l}
0.699318 \\
0.417637)
\end{array}\right.
$$

yields

$$
\xi_{1}=0.601446, \xi_{2}=0.09787 .
$$

The coefficients $b_{j}, j=1,2$, in (16) are determined by (19)

$$
b_{1}=\frac{\xi_{1}}{e^{-\lambda_{1} \Delta}-1}=-1.894427, b_{2}=\frac{\xi_{2}}{e^{-\hat{n}_{2} \Delta}-1}=-0.105573
$$

Hence, the steady state displacement $b_{0}=x_{n}(t \rightarrow \infty)$ is by (17)

$$
b_{0}=-b_{1}-b_{2}=2 \text {. }
$$

The polynomial (24) is therefore 


$$
\begin{aligned}
P(s) & =b_{0}\left(s+\lambda_{1}\right)\left(s+\lambda_{2}\right)+b_{1} s\left(s+\lambda_{2}\right)+b_{2} s\left(s+\lambda_{1}\right) \\
& =\bar{b}_{v} s\left(\dot{\lambda}_{1}+\lambda_{2}\right)+b_{0} \lambda_{1} \lambda_{2}+\bar{b}_{1} s \dot{\lambda}_{2}+b_{2} s \lambda_{1} \\
& =\eta\left(s+\mu_{1}\right),
\end{aligned}
$$

by virtue of (17), and hence

$$
\mu_{1}=\frac{b_{0} \lambda_{1} \lambda_{2}}{b_{0}\left(\lambda_{1}+\lambda_{2}\right)+b_{1} \lambda_{2}+b_{2} \lambda_{1}}=2.000000
$$

As expected the interlacing property $\lambda_{1}<\mu_{1}<\lambda_{2}$ holds.

At this stage the data $\lambda_{1}, \lambda_{2}, \mu_{1}$ and $b_{0}$ have been evaluated. They determine the physical parameters $\gamma_{1}, \gamma_{2}$. $\kappa_{1}$ and $\kappa_{2}$ of the model (1)-(3) as described in Section 4. The elements in the last row of the normalized eigenvector matrix can be determined by (36)

$$
v_{21}=\sqrt{\frac{\lambda_{1}-\mu_{1}}{\lambda_{1}-\lambda_{2}}}=0.850651, v_{22}=\sqrt{\frac{\hat{n}_{2}-\mu_{1}}{\lambda_{2}-\lambda_{1}}}=0.525731
$$

Then $\alpha_{2}$ and $\beta_{2}$ are obtained by (38) and (40)

$$
\begin{aligned}
& \alpha_{2}=\lambda_{1} v \frac{2}{21}+\lambda_{2} v_{22}^{2}=1.000000 \\
& \beta_{1}=-\sqrt{\left(\alpha_{2}-\lambda_{1}\right)^{2} v_{21}^{2}+\left(\alpha_{2}-\lambda_{2}\right)^{2} v_{22}^{2}}=-1.000000 .
\end{aligned}
$$

The elements of the first row of $\mathbf{V}$ are then found by (37)

$$
v_{11}=\frac{\left(\lambda_{1}-\alpha_{2}\right) v_{21}}{\beta_{1}}=0.525731, v_{12}=\frac{\left(\lambda_{2}-\alpha_{2}\right) \nu_{22}}{\beta_{1}}=-0.850651 \text {, }
$$

and by (41)

$$
\alpha_{1}=\lambda_{1} v_{11}^{2}+\lambda_{2} v_{12}^{2}=2.000000 .
$$

The matrix A has thus been fully recovered,

$$
A=\left[\begin{array}{rr}
2 & -1 \\
-1 & 1
\end{array}\right]
$$


In order to extract $\mathbf{K}$ and $\mathbf{G}$ from $\mathbf{A}$ we first solve (45)

$$
\left[\begin{array}{rr}
2 & -1 \\
-1 & 1
\end{array}\right]\left(\begin{array}{l}
y_{1} \\
y_{2}
\end{array}\right)=\left(\begin{array}{l}
1 \\
0
\end{array}\right)
$$

and obtain

$$
y_{1}=1, y_{2}=1 \text {. }
$$

So by (48)

$$
\mathrm{Y}=\left[\begin{array}{ll}
1 & 0 \\
0 & 1
\end{array}\right]
$$

We then determine from (51)

$$
\frac{\sqrt{\gamma_{1}}}{\kappa_{1}}=\sqrt{\frac{2}{\left(\begin{array}{ll}
0 & 1
\end{array}\right)\left[\begin{array}{ll}
1 & 1 \\
1 & 2
\end{array}\right]\left(\begin{array}{l}
0 \\
1
\end{array}\right)}}=1
$$

from which, using (47), we find

$$
\mathbf{K}=\mathbf{A}=\left[\begin{array}{rr}
2 & -1 \\
-1 & 1
\end{array}\right],
$$

and from (46)

$$
\mathbf{G}=\left[\begin{array}{ll}
1 & 0 \\
0 & 1
\end{array}\right] .
$$

The unique system, which produces the data, is shown in Figure 2.

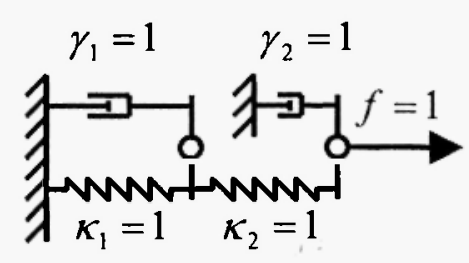

Fig. 2: The reconstructed system 
Note that the strict inequalities $\kappa_{j}>0$ and $\gamma_{j}>0$ that we imposed on the physical parameters of the system are crucial for having a possible unique reconstruction. For example, if all damper coefficients $\gamma_{1}>0$ then the response of the system is discontinuous

$$
x_{n}= \begin{cases}0, & t<0 \\ d, & t>0\end{cases}
$$

where $d-1 /{ }^{K}{ }_{e q}$ is constant and ${ }^{K}{ }_{e q}$ is an equivalent spring constant

$$
\kappa_{e q}=\frac{1}{\sum_{i=1}^{n} \kappa_{i}^{-1}} .
$$

Clearly, there are many spring combinations, which lead to the same value of $\kappa_{e q}$, and hence unique reconstruction is not possible for this case. Similarly if $\kappa_{\ddot{i n}}=0$ then the velocity is constant and the response of the right end of the chain is

$$
x_{n}=\frac{t}{c}
$$

independent of the $2 n-2$ parameters $\kappa_{1}>0$ and $\gamma_{1}>0, \lambda_{j}, j=1,2, \ldots, n-1$.

\section{CONCLUDING REMARKS}

We have shown that the physical elements of an $n$ degrees of freedom chain of springs and dampers may be determined from dynamic measurement of $2 n$ displacements of the chain's end, caused by a static unit load applied to it. If the data are sympathetic with the model, i.e. produced by spring-damper chain, then the reconstruction is unique. This fact follows from the two processes applied, exponential fit and Jacobi matrix reconstruction, each yielding a unique solution. Note that generally a complex exponential fit leads to a nonunique solution due to frequency aliasing. In our case the exponential coefficients are real which allows unambiguous identification.

The analysis has been done for a noise free case. In the present of noise more than $2 n$ response data could be measured and the system may be identified in a least square sense by extending the system of (21) to include $p>n$ equations. The model order can in this case be determine by considering the numerical rank of the over-determined system of equations as described in Braun and Ram, 1987. 


\section{REFERENCES}

C. de Boor and G.H. Golub, 1978, The numerically stable reconstruction of a Jacobi matrix from spectral data, Linear Algebra and Its Application, Vol. 21, pp. 245-260, 1978

S.G. Braun and Y.M. Ram, 1987, Structural parameter identification in the frequency domain: The use of overdetermined systems, ASME Journal of Dynamic Systems, Measurement and Control, Vol. 109, pp. 120-123, 1987

F.P. Gantmakher and M.G. Krein, 1961, Oscillation Matrices and Kernels and Small Vibration of Mechanical Systems, State Publishing House for Technical-Theoretical Literature, Moscow-Leningrad, (Translation: US Atomic Energy Commission, Washington DC)

G.H. Golub and C.F. Van Loan, 1986, Matrix Computations, Johns Hopkins University Press, Baltimore

R.W. Hamming, 1987, Numerical Methods for Scientists and Engineers, Dover Publications, New York

\section{ACKNOWLEDGEMENTS}

The research of the authors was supported in part by a LaSPACE / NASA Space Grant Program, Active InFlight Damage of Fuselage and Remaining Life Assessment.

The research of Y.M. Ram was also supported in part by an NSF grant CMS 9978786, program director A. Flatau. 
